Bioetnia Volumen 12, 2015

\title{
Estudio comparativo de las poblaciones cativo (Prioria copaifera) en el delta del río Atrato
}

\section{Comparative study of the cative populations (Prioria copaifera) in the Atrato river delta}

Robinson Stewart Mosquera M. ${ }^{1}$, Jair Cuesta-Nagles ${ }^{2}$

\section{Resumen}

Se estudió la densidad poblacional y distribución espacial del cativo (Prioria copaifera) en zonas inundables de tres localidades del norte del departamento del Chocó, Colombia. Con el objeto de conocer el estado actual de las poblaciones de la especie para su incorporación a la estructura ecológica principal del Chocó Biogeográfico, se establecieron 14 unidades de muestreo de 50x50m (3,5 ha) distribuidas en los municipios de Carmen de Darién (1,25 ha), Riosucio (1,25 ha) y Unguía (1 ha), donde se censaron los individuos en sus diferentes estadios de crecimiento fustal, brinzal y latizal. Se registró un total de 1589 individuos de P. copaifera en diferentes etapas de desarrollo, siendo el estado fustal el más representativo con 956 (44\%) individuos, seguido por brinzal 338 (31\%) individuos y latizal 295 individuos (25\%). Por otro lado, de las tres localidades estudiadas, el municipio de Riosucio fue quien registró el mayor número de individuos en estado fustal (445), lo que evidencia que la especie en esta localidad no es la que presenta en la actualidad el mayor aprovechamiento por parte de la comunidad y las grandes empresas. A pesar de que la distribución de la estructura vertical de la población muestra un aumento en el estadio fustal, solo $30 \%$ de estos árboles alcanzan la edad reproductiva, esto se debe posiblemente, a que los pobladores no cumplen o desconocen las tallas mínimas para el aprovechamiento de la especie.

Palabras clave: Densidad poblacional, Distribución espacial, Especies amenazadas, Especies asociadas, Estado sucesional.

\section{Abastract}

The population density and spatial distribution of the Captive (Prioria copaifera) were studied in flooded areas of three lo-

\author{
Ingeniero Agroforestal, Investigador Ins- \\ tituto de Investigaciones Ambientales del \\ Pacífico (IIAP), Quibdó, Colombia. \\ e-mail: rmosquera@iiap.org.co \\ Biólogo, contratista Instituto de Investi- \\ gaciones Ambientales del Pacífico (IIAP), \\ Quibdó, Colombia. \\ e-mail: Jcnagles@hotmail.com
}


calities in the north of the department of Chocó, Colombia. In order to know the presentstate of the populations of the species, for its incorporation to the main ecological structure of the Chocó Biogeografico, for this, 14 sampling units of $50 \times 50 m(3.5$ ha) distributed in the municipalities of Carmen de Darién (1.25 ha), Riosucio (1.25 ha) and Unguia (1 ha), within which individuals were recorded in their different stages of high forest, seedling and polewood. A total of 1589 individuals of $P$. copaifera were recorded at different stages of development. The fustal state was the most representative with 956 (44\%) individuals, of the total sample, followed by 338 (31\%) individuals and latizal 295 (25\%) individuals. On the other hand of the three localities studied, the municipality of Riosucio was the one that registered the largest number of individuals in a fustal state 445, which shows that the species in this locality is not the one that currently presents the greatest use by the community and large companies. Although the distribution of the vertical structure of the population shows an increase in the fustal stage, only $30 \%$ of the trees of the population in this stage reach the reproductive age, this is possibly because the villagers do not respect or they do not know the minimum sizes for the use of the species.

Keywords: Associated species, Population density, Spatial distribution, Successional status, Threatened species.

\section{Introducción}

Uno de los problemas ambientales más relevantes a escala global, es la reducción de la diversidad biológica asociada con los efectos de pérdida, fragmentación y transformación del hábitat como consecuencia de las actividades humanas (Hanken 1999, Knutson et al. 1999, 2000). Muchas poblaciones, confinadas en bosques fragmentados, desaparecen con el paso del tiempo porque no alcanzan a mantener una población mínima viable. La persistencia de las poblaciones en tales casos depende de las características intrínsecas de la especie y es asociada con las condiciones ambientales y el tamaño del bosque o fragmento (Robinson y Ramírez 1982, Lovejoy et al. 1986, Collins y Storfer 2003, Pounds et al. 2006).

En Colombia no existe una ordenación forestal sostenible de los bosques naturales a pesar de que cubren cerca del $50 \%$ de su territorio continental (más de 50 millones de hectáreas). La pérdida de especies arbóreas es una amenaza a la diversidad biológica, siendo la deforestación, incendios forestales, crecimiento demográfico y el cambio de uso del suelo, las principales causas de pérdida de biodiversidad en los países tropicales. Un tipo de bosque especialmente importante en Colombia es el catival; este bosque se desarrolla en las llanuras periódicamente inundables de los ríos Atrato y León, departamentos Chocó y Antioquia, que vierten sus aguas al golfo de Urabá, Darién colombiano. Los cativales sobresalen por conformar comunidades de varias especies de árboles entre las cuales el cativo ( $P$. copaifera Grisebach) es dominante. Allí se encuentran los cativales más extensos existentes en América, su área original cubría 363.000 ha (González et al. 1991). Por más de 70 años el cativo ha sido la base principal de la industria maderera en el bajo Atrato. Los cativales se clasifican de acuerdo con la duración del período de inundación; en tipo A1, el de mayor duración de la inundación (más de seis meses), Catival A2, permanece inundado entre tres y seis meses y Catival A3 permanece inundado menos de tres meses al año (González et al. 1991). La dominancia del cativo decrece desde el tipo A1 al A3, dando como resultado una masa relativamente homogénea de especies arbóreas de alta producción maderera. No existen estudios que establezcan el estado poblacional de las especies. Esto no permite desarrollar estrategias de conservación y de alguna manera contribuye a la extinción de sus poblaciones, ecológicamente 


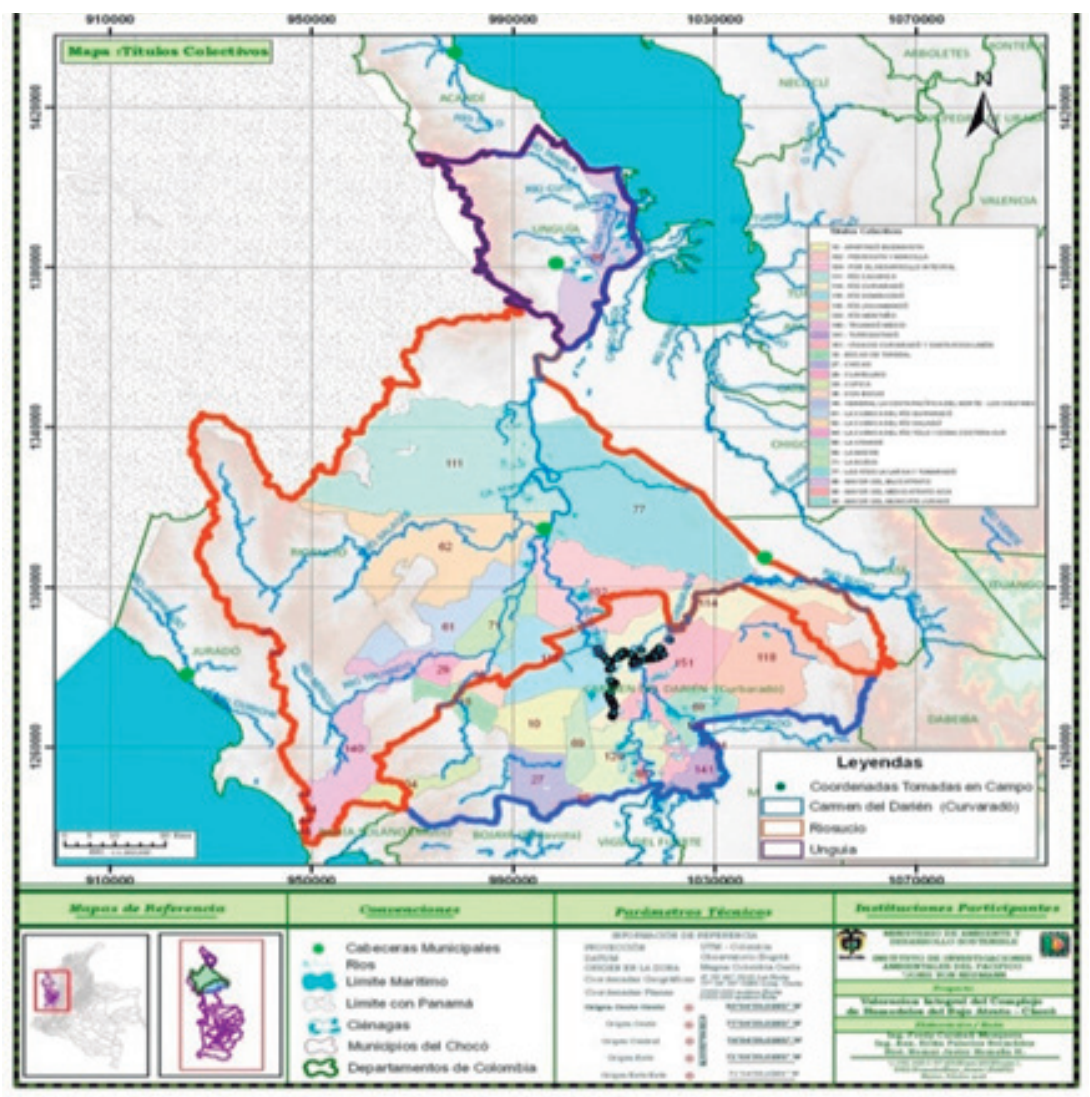

Figura 1. Ubicación geográfica de la zona de estudio (Carmen de Darién, Riosucio y Unguía).

importantes en términos de conservación.

Area de estudio. El estudio se realizó en tres localidades al norte del departamento del Chocó (Figura 1):

Carmen del Darién. Se encuentra ubicado a la margen derecha del río A trato en la eco-región del Urabá chocoano a los $7^{\circ}$ 9'28' norte y $76^{\circ} 58^{\prime} 15^{\prime}$ " oeste, a $4 \mathrm{~m}$ de elevación y una precipitación promedio de $7.774 \mathrm{~mm}$ (Poveda et al. 2004); dista de Quibdó, capital del departamento de Chocó, a $369 \mathrm{~km}$ por vía fluvial; su cabecera municipal es Carburado con una superficie de $4.700 \mathrm{~km}^{2}$; sus suelos son pantanosos de la planicie aluvial del río Atrato, característico de esta zona del Chocó biográfico, que se extiende desde Vigía del Fuerte hasta el golfo de Urabá.

Riosucio, se encuentra ubicado en la zona noroccidental del departamento del Chocó, $7^{\circ} 26^{\prime}$ 26 " de latitud norte y $77^{\circ} 07^{\prime} 08^{\prime \prime}$ oeste; hace parte del denominado Darién colombiano, a 4 $\mathrm{m}$ de elevación y una precipitación promedio de $7.774 \mathrm{~mm}$ (Poveda et al. 2004); pertenece a la llanura aluvial.

Unguía se localiza en el eco-región del Urabá chocoano, al noroccidente del departamento del Chocó, en las coordenadas $8^{\circ} 1$ ' $30^{\prime}$ " norte y $77^{\circ}$ 09' 25" occidental; su cabecera municipal se encuentra a $478 \mathrm{~km}$ de distancia de Quibdó por vía fluvial y una altura de $5 \mathrm{~m}$ sobre el nivel del mar (Plan de Desarrollo Municipio 2008-2011).

\section{Métodos}

Muestreo de las poblaciones de cativo. La densidad poblacional de Prioria copaifera en los sitios de muestro (Carmen del Darién, Riosucio y Unguía) se realizó en zonas aluviales y áreas influidas por humedales con diferentes niveles de 
intervención antrópica (intervenidas, semi-intervenidas y sin intervención antrópica) mediante recorridos sistemáticamente al azar partiendo que la distancia mínima entre los transectos fuera de $100 \mathrm{~m}$, evitando de esta manera que se sobrelaparan. En Carmen de Darién y Riosucio se realizaron cinco transectos de 50x50 m cada uno $\left(12.500 \mathrm{~m}^{2}\right)$ para un área total de 2,5 ha. En Unguía se establecieron cuatro transeptos de 50x50m equivalentes a 1 ha, dentro de los cuales se censaron todos los individuos de la especie en sus diferentes estados de desarrollo: plántulas $(0,31 \mathrm{~cm}$ a $1,5 \mathrm{~m})$, juveniles $(>1,5 \mathrm{~m}$ de altura $\mathrm{y}$ DAP $\leq 9,9 \mathrm{~cm})$ y adultos ( $\geq 10 \mathrm{~cm}$ DAP); de igual forma se registraron las alturas total y comercial. (Gentry 1993), además se registró la vegetación asociada con $P$. copaifera con el objeto de entender la importancia ecológica de las especies que interactúan en estos ecosistemas.

Análisis de los datos. Este se realizó teniendo en cuenta la importancia de la especie a partir de la utilización del índice de valor de importancia (IVI) que se calculó de acuerdo con Villareal et al. (2004) a través de la fórmula IVI $=\mathrm{Ar}+\mathrm{Fr}$, donde Ar es la abundancia relativa de la especie y Fr la frecuencia relativa de la especie; de igual forma se calculó el número de árboles por $\mathrm{Ha}$ expresado a través de la fórmula:

\section{$\mathrm{Na} / \mathrm{Ha}=1 /(\mathrm{Np})^{*}(\mathrm{Tp})^{*} \mathrm{Ne}$}

donde $\mathrm{Na} / \mathrm{Ha}$ es número de árboles por $\mathrm{Ha}$, $\mathrm{Np}$ es el número de parcelas muestreadas, Tp es el tamaño de las parcelas expresado en Ha y $\mathrm{Ne}$ número de árboles encontrados durante el inventario, partiendo de que las áreas de muestreo tomadas en cada localidad fueron diferentes ( 1.25 ha para Carmen de Darién y Riosucio y una ha para Unguía).

Se tomó una unidad base de 0.1 ha para realizar análisis comparativos de las variables (abundancia y densidad) entre las mismas y con otros estudios en el trópico.

\section{Resultados y discusión}

Densidad y abundancia poblacional del cativo. Analizada la estructura de los relictos boscosos de cativo en las 14 unidades de muestreo, se reporta un total de 1.589 individuos de $P$. copaifera en diferentes estadíos de crecimiento (fustales, brinzal, latizal). El estado fustal es el más representativo con 956 individuos (Figura 2), destacándose el municipio de Riosucio como el que presenta el mayor número de individuos (445, 47\%) (71,2 ind/ha), seguido de Carmen de Darién con 306 (32\%) individuos (48,96 ind/ ha); por último en el municipio de Unguía se registraron 205 (21\%) individuos (51,25 ind/ha).

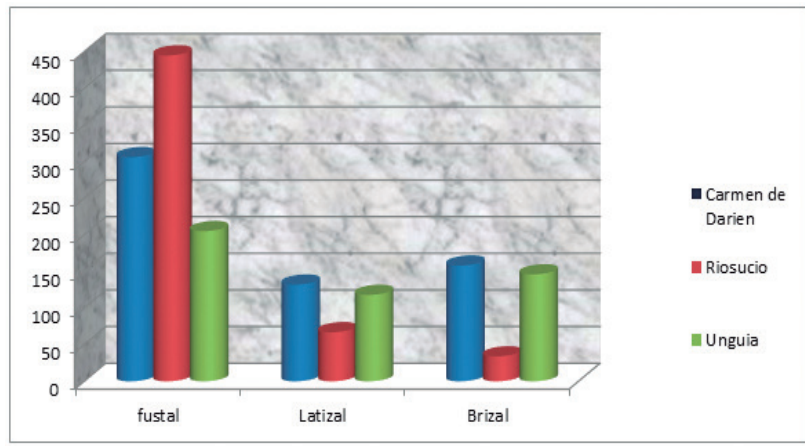

Figura 2. Estado sucesional de Prioria copaifera (cativo) en las diferentes localidades

A pesar de que la distribución de la estructura vertical de la población muestra un aumento en el estadío fustal, solo 50 árboles de la población en este estadío alcanzan la edad reproductiva. Lo anterior se corroboró con los datos obtenidos por Conif y Pizano (1999) quienes revelan que el cativo adquiere la edad biológica reproductiva a los $40 \mathrm{~cm}$ de diámetro y la edad silvicultural o de aprovechamiento a los $60 \mathrm{~cm}$ de DAP. E1 comportamiento de esta población puede estar relacionado con las estrategias de lento crecimiento de la especie pues según los argumentos de Condit (1995) y Giraldo y del Valle (2011) esta especie puede tardar más de 130 años en alcanzar el diámetro comercial, es decir de pasar de $1 \mathrm{~cm}$ de diámetro a $60 \mathrm{~cm}$ de DAP que es el diámetro 


\section{Bioetnia Volumen 12, 2015}

apto para el aprovechamiento, porque la especie en condiciones naturales obtiene un incremento dimétrico anual de aproximadamente $0,31 \mathrm{~cm}$ al año, es decir que en 90 años puede alcanzar 40 cm de DAP (Giraldo y del Valle 2011). Además de lo anterior, esta especie necesita unos requerimientos para su buen desarrollo.

Situación contraria se pudo observar en los sitios de muestreo, donde se evidenció el constante aprovechamiento por parte de los pobladores locales de la especie en diámetros inferiores a los permitidos, situación que produjo cambios abruptos en la estructura de la población, pues $P$. copaifera una especie esciófita, que necesita condiciones mínimas de luminosidad para su resiliencia; el constante aprovechamiento de madera en el remante puede estar infiriendo en el reclutamiento de la misma e incidiendo de manera directa en la reducción del primer estadío (brinzal), situación que concuerda con lo reportado por Linares (1996) quien manifiesta que la cosecha continua de esta especie causa una abrupta apertura en el dosel y en consecuencia, las plántulas de la regeneración natural temprana y los fustales remanentes con hojas de sombra se ven sometidos a un intempestivo y elevado incremento en la exposición solar y cambios bruscos de temperatura, ocasionando graves pérdidas de población vegetal por mortalidad, así como retraso en su crecimiento y desarrollo, por el estrés fisiológico inducido.

Situación contraria plantean Valverde-Barrantes y Rocha (2014) quienes evaluaron el impacto de la extracción en la estructura y dinámica de la plántulas en un bosque dominado por $P$. copaifera, para lo cual tuvieron en cuenta tres escenarios: un bosque con diferentes grados de transición, el primero de 2 años, seguido de uno de 12 años de aprovechamiento y un remanente sin intervención, concluyendo que tiempo después de la intervención aumenta la composición florística del bosque, lo que explica la baja diversidad de los cativales del Carmen del Darién, comparado con los de la zona baja del Atrato. Por otro lado, estos mismos autores consideran que en el remante boscoso intervenido hay mayor densidad de plántulas, sin embargo, este mismo remanente presentó la mayor mortalidad de plántulas en este estadío, demostrando así la capacidad de la especie de mantenerse aun en condiciones adversas.

Las poblaciones de $P$. copaifera presenta un alto potencial de regeneración continuo en dichas condiciones ambientales, también, debido a que la mayor distribución de individuos se encuentra en las clases diamétricas iniciales $(10$ y $40 \mathrm{~cm}$ de DAP), por tal motivo se evidencia que estas zonas están dirigidas a un proceso de conservación porque muestran que se trata de una población joven.

El estado fustal fue dominante en relación con los otros estadíos, teniendo en cuenta que el estado brinzal reportó 338 individuos y latizal 295; a pesar de esto, la especie presenta un alto potencial de regeneración y la baja presencia de estados juveniles se encuentra directamente relacionada con el cambio de coberturas, las cuales han sido drásticamente transformados por la extracción de madera.

Estructura vertical. De acuerdo con lo observado en los distintos sitios de muestreo, se pudieron distinguir 3 estratos los cuales estaban bien definidos: brinzal, latizal y fustal; este último se subdividió en alto (dominante), medio (co-dominante) y bajo (dominado), dentro de los cuales el estrato alto presentó 49 (6\%) individuos con alturas $\leq 23 \mathrm{~m}$, mientras que en el estrato medio se agruparon la mayor parte de los individuos (538) los cuales se encuentran entre 13 y $19 \mathrm{~m}$ con el $61 \%$ del total de los individuos registrados. En el estrato arbóreo bajo (dominado) constituido por individuos con alturas entre 5 y $12 \mathrm{~m}$ solo se registraron 292 (33\%) individuos.

La altura vertical del bosque presentó correlación directa con respecto a las clases diamétricas, a raíz de que la mayoría de los individuos registrados están concentrados en los primeros estratos. Lo anterior, deja manifiesto que los bosques de 
cativo en las áreas inundables del delta del río Atrato se encuentra en proceso de recuperación, esto posiblemente se debe a que parte del bosque fue transformado para uso diferente a la madera y lo poco que queda se ha dejado como remanente, lo que coincide con los resultados obtenidos por IIAP(2013), Asprilla etal. (2003) y Sanín y Duque (2006) quienes en investigaciones realizadas en los bosques del departamento del Cauca, Colombia, determinaron que la mayoría de los individuos se encuentran en proceso de crecimiento (Tabla 1).

Tabla 1. Estructura vertical de las poblaciones de cativo

\begin{tabular}{lccc}
\hline & $\begin{array}{c}\text { Clases } \\
\text { de alturas }\end{array}$ & Rango & $\begin{array}{c}\mathbf{N}^{\circ} \text { de } \\
\text { ind }\end{array}$ \\
\hline Bajo (dominado) & $\mathrm{I}$ & $05-12$ & 292 \\
Medio (codominante) & $\mathrm{II}$ & $13-19$ & 538 \\
Alto (dominante) & $\mathrm{III}$ & $>20$ & 49 \\
\hline
\end{tabular}

De acuerdo con lo observado, la altura promedio que poseen los árboles de $P$. copaifera registrados en las diferentes parcelas, fue de 14 $\mathrm{m}$ factor que se relaciona con los altos niveles de intervención que presenta el bosque. Por consiguiente, las especies que ocupan los mayores estratos fueron aquellas que durante épocas de deforestación se dejaron de lado, ya sea porque al momento del aprovechamiento no contaban con los diámetros requeridos por los extractores o porque presentaban malformaciones en su estructura (huecos, hongos), generando cambios notables en la dinámica del bosque, lo cual, repercute en el ecosistema y se manifiesta a través de cambios en la dieta alimenticia de algunos grupos faunísticos, la etología y reproducción de algunos organismos.

Distribución horizontal. De los 1.589 individuos registrados para fustales, 812 se ubican en las tres primeras categorías diamétricas: I $(10,0-$ 24,6) con 343 individuos, II (24,6-39.2), 296 y III $(39,2-53,8) 173$ individuos respectivamente; la clase I con $37 \%$ posee la mayor cantidad de los individuos, los 124 individuos restantes corresponden a las categorías IV $(53,8-68,4)$ y V $(\geq 70)$ lo que deja de manifiesto que los ecosistemas inventariados corresponden a un bosque en proceso de recuperación, resultados que coinciden con los de Arruda et al. (2011) y Hernández-Stefanoni et al. (2011) quienes sostienen que la comunidad vegetal se encuentra en coherente proceso de desarrollo en dirección a etapas de crecimiento y productividad vegetal más avanzados, una vez que se confirma la existencia de abundantes individuos jóvenes que irán a suceder individuos arbóreos pertenecientes a las clases diamétricas superiores.

En lo relacionado con las parcelas analizadas en los relictos de cativo del municipio de Unguia se puede observar que el bosque se encuentra en estado sucecional esto a raíz de que el mayor número de individuos se encuentra en las clases diamétricas inferiores tendiendo a formar la "J" invertida, lo que confirma el grado de recuperación del ecosistema, no obstante, se observaron algunas excepciones en individuos de P. copaifera, donde se evidenciaron malformaciones (bifurcaciones y ahoyamientos en el fuste), hecho que se atribuye a las condiciones edáficas, ausencia de manejo (podas) e intervenciones antrópicas como tala selectivas, las cuales generan claros. Estos resultados concuerdan con los obtenidos por la Restrepo et al. (2012) quienes determinaron que en los bosques primarios las especies esciófitas presentan patrones de atrofismo, mientras que las heliófitas en forma recta.

Por otro lado Brokaw (1985) sostiene que la ausencia de reclutamiento de plantas en estados juveniles se debe a la falta de condiciones de luminosidad en el sitio, concordando con las condiciones observadas en el sitio de estudio, situación que dificulta las condiciones de regeneración de la especie.

Una consideración importante en el catival, es el tamaño de las aperturas en el dosel causadas 


\section{Bioetnia Volumen 12, 2015}

por el aprovechamiento. Si las aperturas son demasiado grandes, el sitio se secará y llegaría a ser más favorable para otras especies en lugar de P. copaifera. Debido a la característica de homogeneidad del estrato superior de los cativales, lo más importante es mantener al máximo posible la estructura del bosque; controlando la intensidad del aprovechamiento, se mantiene un ambiente favorable para la germinación de semillas y el desarrollo de la regeneración natural del cativo (Grauel y Pineda 2001). La alta densidad (individuos/ha) puede facilitar la implementación de técnicas silviculturales y la posibilidad de proponer planes de manejo para la conservación y aprovechamiento sostenible (López y Montero 2007). Es debido a los cambios extremos que a causa de la intervención antrópica se genera desplazamiento de especies de fauna a áreas distantes para la búsqueda de recursos energéticos que les permitan realizar sus funciones metabólicas.

\section{Caracterización ecológica del ecosistema de catival}

Especies asociadas con el cativo. En el reconocimiento de la zona de estudio, se encontró una relativa riqueza de especies arbóreas en contraposición con la característica, neotrópica del bosque entorno al $P$. copaifera al igual que las observaciones detalladas del ecosistema qu e brindan los elementos para definir la distribución de la especie de acuerdo con su asociación en catival homogéneo o puro y catival mezclado. Se registran 1.790 individuos distribuidos en las tres localidades estudiadas, pertenecientes a 35 especies de 15 familias, donde predominan Anacardiaceae con Anacardium excelsum, Spondias mombim; Arecaceae con Euterpe cuatrecasana, Raphia taedigera; y Cluciaceae con Symphonia globurifera, Callophilum mariae. Existe variedad de especies arbóreas de interés comercial las cuales poseen gran número de individuos, dentro de los cuales se destaca el Prioria copaifera (102.88 ind/ $\mathrm{ha}^{-1}$ ) seguida del caracolí (Anacardium excelsum), machare (Symphonia globulifera) y sande (Brosimun utile) con 13 individuos cada uno (Tabla 1). Las principales asociaciones están representadas en mayor proporción por especies como Cecropia $s p$. (yarumo), Erythrina sp. (chachafruto), Inga sp. (guamos), Pachira sp. (salero), Polygonum acuminatum, Thalia geniculata, Hymenachne amplexicaulis, Cyperus ligulae, Sesbania sp., Ipomeae sp., Phaseolus campestre, sumadas a una serie de arbustivas y rastreras entre las cuales aparecen ejemplares aislados de $M$. arborescens, T. tahedijera restringidas a las zonas de apertura del sotobosque con acceso de luz, indispensable para su permanencia (Tabla 2).

La alta diversidad de taxas y la variedad de formas botánicas (lianas, hierbas, bejucos, plantas del piso, arbolitos, arbustos y palmas) predominan especies como baba de zaino (Dieffenbachia longispatha), heliconia (Heliconia brachiantha) las cuales ocupan grandes áreas abiertas en los cativales la "pita" (Aechmea magdalenense) y abundan los helechos, como las Dryopteridoidea "H. Hurtado" (Tectaria incisa) entre otros.

\section{Consideraciones finales}

Las asociaciones de cativo presentes a lo largo del delta del río Atrato presentan cierta tendencia a la heterogeneidad, debido posiblemente, a que se desarrolla en manchas homogéneas y por la intervención humana en la zona.

Los altos niveles de intervención antrópica asociado con los sitios de muestreo influyen negativamente en las poblaciones de cativo porque en condiciones normales la especie es dominante en los tres estratos del bosque. Con base en los datos registrados en el municipio de Unguía se pudo evidenciar que la especie presenta pocos individuos, debido al cambio del uso del suelo para establecimiento de cultivos y ganadería extensiva.

La baja representatividad de los individuos en el estrato inferior, se atribuye a las condiciones 
Poblaciones cativo en el delta del río Atrato. RS Mosquera, J Cuesta-Nagles

Tabla 2. Especies asociadas con el cativo

\begin{tabular}{|c|c|c|c|c|c|}
\hline $\mathbf{N}^{\circ}$ & Especies & Nombre científico & Familia & $\mathrm{N}^{\circ}$ de árboles & Área basal \\
\hline 1 & Aceite María & Callophilum mariae & Cluciaceae & 24 & 7,87 \\
\hline 2 & Arroz con coco & Andira inermis & Papilonacea & 2 & 0,47 \\
\hline 3 & Arracacho & Montrichardia arborescens & Aracacea & 1 & 0,06 \\
\hline 4 & Aserrín & Parkia pendula & Fabaceae & 30 & 9.68 \\
\hline 5 & Bambudo & Ptocarpus oficinalis & Fabaceae & 10 & 3,27 \\
\hline 6 & Caracolí & Anacardium excelsum & Anacardiaceae & 26 & 4,83 \\
\hline 7 & Carbonero & Licania arbóreo & Chrysobalanaceae & 18 & 1.01 \\
\hline 8 & Cativo & Prioria copaifera & Caesalpinaceae & 1035 & 114,48 \\
\hline 9 & Capitancillo & $n n$ & $\mathrm{Nn}$ & 4 & 1,00 \\
\hline 10 & Cuero negro & Hyrtella racemosa & Chrysobalanacea & 5 & 1,44 \\
\hline 11 & Cucharo & Swartzia sp & Papilonacea & 3 & 0,58 \\
\hline 12 & Guamo & Inga sp & Fabaceae & 53 & 3,98 \\
\hline 13 & Guacimo loro & Inga sp & Tiliácea & 3 & 0,86 \\
\hline 14 & Guacimo baba & Apeiba tibourbou & Tiliaceae & 2 & 0,55 \\
\hline 15 & Guacimo & Inga sp & Tiliaceae & 3 & 0,59 \\
\hline 16 & Guasco & Couratari sp & Lecythidaceae & 1 & 0,09 \\
\hline 17 & Guino & Carapa guianensis & Meliaceae & 5 & 0,52 \\
\hline 18 & Hobo & Spondias mombim & Anacardiaceae & 17 & 1,47 \\
\hline 19 & Lirio & Crouma macrocarpa & Apocynaceae & 1 & 0,11 \\
\hline 20 & Machare & Symphonia globurifera & Cluciaceae & 22 & 1,48 \\
\hline 21 & Mangle & Rapanea sp & Myrcinacea & 5 & 1,94 \\
\hline 22 & Mano de guagua & $n n$ & $\mathrm{Nn}$ & 1 & 0,20 \\
\hline 23 & Paco & Cespedesia macrophylla & Ochnaceae & 9 & 1,13 \\
\hline 24 & Paco guitarro & Alseis blacakiana & Rubuaceae & 1 & 0,17 \\
\hline 25 & Palma chunga & Standleyanum I.h & Aracaceae & 7 & 1,18 \\
\hline 26 & Palma murrapo & Euterpe cuatrecasana & Arecaceae & 19 & 0,93 \\
\hline 27 & Palma pangana & Raphia taedigera & Arecaceae & 20 & 0,77 \\
\hline 28 & Peine mono & Apeiba aspera & Tiliaceae & 10 & 2,20 \\
\hline 29 & Roble & Tabebuia rocea & Bignoniaceae & 9 & 0,64 \\
\hline 30 & Salero & lecythis sp & Lecytdaceae & 25 & 4,01 \\
\hline 31 & Sande & Brosimun utile & Moraceae & 26 & 2,03 \\
\hline 32 & Sietecuero & Tibouchina lepidota & Melastomataceae & 9 & 3,39 \\
\hline 33 & Sueldo & Ficus dendrata & Moraceae & 6 & 1,67 \\
\hline 34 & Taparo & Crescetia cujete L. & Bignonaceae & 1 & 0,47 \\
\hline 35 & Yarumo & Cecropia sp & Cecropiaceae & 24 & 3,77 \\
\hline Total & & & & 1437 & 10.69 \\
\hline
\end{tabular}

edáficas del lugar, la alta concentración de necromasa en el sotobosque y la ruptura en los procesos ecológicos naturales por parte de las actividades de los pobladores circundantes a estos ecosistemas.

\section{Literatura citada}

Alcaldía de Unguía. Plan de Desarrollo Municipal. 20082011. 


\section{Bioetnia Volumen 12, 2015}

Arruda DM, Brandao DO, Costa FV, Tolentino GS, Brasil RD, D'Angeloneto SN, Nunes-Ferreira YR. 2011. Structural aspects and fl oristic similarity amongtropical dry forest fragments with different management histories in Northern Minas Gerais, Brazil. Rev Arvore. 35: 131-42.

AsprillaA, Mosquera C, Valoyes H, Cuesta H, García F 2003. Composición florística de un bosque pluvial tropical (bp-T) en la Parcela Permanente de Investigación en Biodiversidad (PPIB) en Salero, Unión Panamericana, Chocó. En: García F, Ramos YA, Palacios Lloreda J, Arroyo JE, Mena Marmolejo A, Maribel GA (eds.). Salero. Diversidad biológica de un bosque pluvial tropical (bp-T); p. 39-44.

Brokaw N. 1985. Treefall, regrowth and community structure in tropical forests. pp. 53-71. In: Pickett S, White $\mathrm{P}$ (eds.). The ecology of natural disturbances and dynamics. New York: Academic Press Inc.

Collins J, Storfer A. 2003. Global amphibian declines: Sorting the hypotheses. Divers Distrib. 9: 89-98. URL disponible en: http://onlinelibrary.wiley.com/ doi/10.1046/j.1472-4642.2003.00012.x/pdf

Conif, Corporación Nacional de Investigación y Fomento Forestal \& Pizano. 1999. Manejo y evaluación del ecosistema del Catival. Serie técnica $N^{\circ} 44$. Bogotá: Conif y Pizano SA; 118 pp.

Condit R. 1995. Research in large, long-term tropical forest plots. Trends Ecol Evol. 10 (1): 18-22. URL disponible en: https://www.ncbi.nlm.nih.gov/pubmed/21236939

Gentry AH. 1993. Riqueza de especies del Pacífico. In: Leyva P (ed.). Colombia Pacífico II. Bogotá: Fondo FEN. URL disponible en: http://www.banrepcultural. org/blaavirtual/geografia/cpacifi2/indicecpacific2.htm

Giraldo JA, del Valle JI. 2011. Estudio del crecimiento de Prioria copaifera (Caesalpinaceae) mediante técnicas dendrocronológicas. Rev Biol Trop. 54 (4): 1813-31. URL disponible en: http:// www.scielo.sa.cr/scielo.php?script=sci arttext\&pi$\mathrm{d}=\mathrm{S} 0034-77442011000400032$

González H, Gómez HD, Arteaga JF. 1991. Aspectos estructurales de un bosque de cativo en la región del bajo Atrato, Colombia. Rev Fac Nal Agron. 44 (1-2): 3-50. URL disponible en: https://revistas.unal.edu.co/index. php/refame/article/view/28474/28874

Grauel WT, Pineda RM. 2001. Manual técnico para el manejo sostenible de los cativales en Darién, Panamá. Ciudad de Panamá: Autoridad Nacional del Ambiente de Panamá (ANAM). Smithsonian Tropical Research Institute, Organización Internacional de Maderas Tropicales (OIMT); 101 pp.

Hanken J. 1999. Why are there so many new amphibian species when amphibians are declining? Trends Ecol
Evol. 14 (1): 7-8.

Hernández-Stefanoni JL, Dupuy JM, Tun-DzulF, May-Pat F. 2011. Infl uence of landscape structure and stand age on species density and biomass of a tropical dry forest across spatial scales. Landscape Ecol. 26: 355-70.

Instituto de Investigaciones Ambientales del Pacífico(IIAP) y Corporación Autónoma para el Desarrollo Sostenible del Chocó (CODECHOCÓ). 2013. Convenio $N^{\circ} 005$ Interadministrativo, el proyecto «Implementación de estudio base para especies forestales amenazadas en el departamento del Chocó». Quibdó: IIAP, CODECHOCÓ. URL disponible en: http://siatpc.iiap.org.co/ docs/avances/lbefa.pdf

Knutson MG, Sauer JR, Olsen DA, Mossman MJ, Hemesath LM, Lannoo MJ. 1999. Effects of landscape composition y wetland fragmentation on frog y toad abundance y species richness in Iowa y Wisconsin, USA. Conserv Biol. 13 (6): 1437-46.

Knutson M, Sauer JR, Olsen DA, Mossman MJ, Hemesath LM, Lannoo MJ. 2000. Landscape associations of Frog y Toad species in Iowa y Wisconsin, USA. J Iowa Acad Sci. 107 (3): 134-45. URL disponible en: https:// www.umesc.usgs.gov/documents/publications/2000/ knutson_b_landscape_associations 2000.pdf

Linares R. 1996. Caracterización del bosque de cativo (Prioria copaifera) en dos estados sucesionales: clímax y 21 años post-aprovechamientos. Simposio Internacional Posibilidades de Manejo Forestal Sostenible en América Tropical. pp. 26-33. Bogotá: Corporación Nacional de Investigación y Fomento Forestal.

López R, Montero I. 2007. Prioria copaifera. En: Cárdenas D, Salinas NR (eds). Libro Rojo de plantas de Colombia. Especies maderables amenazadas. Vol. 4. Bogotá: Instituto de investigaciones Amazónicas (SINCHI) y Ministerio de Ambiente, Vivienda y Desarrollo Territorial. 234 pp. URL disponible en: https:/www. sinchi.org.co/files/publicaciones/publicaciones/pdf/ LR MADERABLES.pdf

Lovejoy TE, Bierregaard RO, Rylands AB, Malcolm JR, Quintela CE, Harper LH, et al. 1986. Edge and other effects of isolation on Amazon forest fragments. pp. 257-85. In: Soulé ME (ed.). Conservation biology: the science of scarcity and diversity. Sunderland: Sinauer Associates, Inc.

Poveda C, Rojas-P CA, Rudas-Ll A, Rangel-Ch O. 2004. El Chocó Biogeográfico: Ambiente físico. pp. 1-21. En: Rangel-CH JO (ed). Colombia Diversidad Biótica IV: El Chocó Biogeográfico/Costa Pacífica. Bogotá: Instituto de Ciencias Naturales, Universidad Nacional de Colombia. URL disponible en: http:// www.colombiadiversidadbiotica.com/Sitio_web/ LIBROS DEL I AL IV/Entradas/2011/3/28 CO- 
LOMBIA DIVERSIDAD BIOTICA IV.html

Pounds JA, Bustamante MR, Coloma LA. 2006. Widespread amphibian extinctions from epidemic disease driven by global warming. Nature. 439: 161-7.

Restrepo HI, Orrego SA, Galeano OJ. 2012. Estructura de bosques secundarios y rastrojos montanos bajos del norte de Antioquia, Colombia. Rev Colomb Forest. 15 (2): 173-89. URL disponible en: http://revistas.udistrital.edu.co/ojs/index.php/colfor/article/view/3608/5618

Ríos S. 1996. Estudio de la arquitectura de la comunidad de Prioria copaifera Grisebach (Caesalpinaceae) en un bosque inundable de la región del bajo Atrato, Chocó, Colombia. Rev Colomb Forest. 6 (13): 5-37. URLdisponible en: http://revistas.udistrital.edu.co/ojs/ index.php/colfor/rt/printerFriendly/3265/4762+\&c$\mathrm{d}=5 \& \mathrm{hl}=\mathrm{es}-419 \& \mathrm{ct}=\mathrm{clnk} \& \mathrm{gl}=\mathrm{co}$

Robinson JG, Ramírez CJ. 1982. Conservation biology of neotropical primates. pp. 329-44. In: Genoways HH,
Mares MA (eds.). Mammalian biology in South America. Vol. 6. The Pymatuning Symposia on Ecology. Pittsburg: University of Pittsburg.

Sanín D, Duque CA 2006. Estructura y composición florística de dos transeptos localizados en la reserva forestal protectora Río Blanco (Manizales, Caldas, Colombia). Bol Cient Mus Hist Nat U de Caldas. 10: 47-8.

Valverde-Barrantes OJ, Rocha OJ. 2014. Impactos de la tala en la estructura forestal y la dinámica de las plántulas en una selva tropical dominada por Prioria copaifera (Fabaceae) (Talamanca, Costa Rica). Rev Biol Trop. 62 (1): 350 .

Villareal H,Alvarez M, Cordoba S, EscobarF, Fagua G, Gast F, et al. 2004. Manual de métodos para el desarrollo de inventarios de biodiversidad. Bogotá: Programa de Inventarios de Biodiversidad, Instituto de Investigación de Recursos Biológicos Alexander Von Humboldt. 